\title{
Implementing a Sepsis Resuscitation Bundle Improved Clinical Outcome: A Before-and-After Study
}

\author{
Jeongmin Kim, M.D., Sungwon Na, M.D., Ph.D., Young Chul Yoo, M.D., Ph.D., and Shin Ok Koh, M.D., Ph.D. \\ Department of Anesthesiology and Pain Medicine, Yonsei University College of Medicine, Seoul, Korea
}

Background: Unlike other diseases, the management of sepsis has not been fully integrated in our daily practice. The aim of this study was to determine whether repeated training could improve compliance with a 6-h resuscitation bundle in patients with severe sepsis and septic shock.

Methods: Repeated education regarding a sepsis bundle was provided to the intensive care unit and emergency department residents, nurses, and faculties in a single university hospital. The educational program was led by a multidisciplinary team. A total of 175 adult patients with severe sepsis or septic shock were identified ( 88 before and 87 after the educational program). Hemodynamic resuscitation bundle and timely antibiotics administration were measured for all cases and mortality at 28 days after sepsis diagnosis was evaluated. Results: The compliance rate for the sepsis resuscitation bundle before the educational program was poor $(0 \%)$, and repeated training improved it to $80 \%(\mathrm{p}<0.001)$. The 28 -day mortality was significantly lower in the intervention group $(16 \%$ vs. $32 \%, p=0.040)$. Within the intervention group, patients for whom the resuscitation bundle was successfully completed had a significantly lower 28-day mortality than other patients $(11 \%$ vs. $41 \%, \mathrm{p}=0.004)$.

Conclusions: Repeated education led by a multidisciplinary team and interdisciplinary communication improved the compliance rate of the 6-h resuscitation bundle in severe sepsis and septic shock patients. Compliance with the sepsis resuscitation bundle was associated with improved 28-day mortality in the study population.

Key Words: critical pathways; education; interdisciplinary communication; sepsis.

\section{Introduction}

Severe sepsis and septic shock are the leading causes of death in non-cardiac intensive care units (ICU).[1,2] Management of sepsis based on the Surviving Sepsis Campaign (SSC) guidelines[3] has been associated with survival benefits.[4-9] For the management of severe sepsis, the SSC recommended the im-

Received on February 27, 2014 Revised on June 24, 2014

Accepted on August 28, 2014

Correspondence to: Sungwon Na, Department of Anesthesiology and Pain Medicine, Yonsei University College of Medicine, 50-1 Yonsei-ro, Seodaemun-gu, Seoul 120-752, Korea

Tel: +82-2-2228-2420, Fax: +82-2-2227-7897

E-mail: nswksj@yuhs.ac

* No potential conflict of interest relevant to this article was reported. plementation of an early goal-directed sepsis resuscitation bundle. Although the guidelines provide a road map for the care of severe sepsis patients, their success depends on the compliance of clinicians. In previous studies a national training program based on the SSC guidelines improved compliance with recommended processes of care for severe sepsis in ICUs. However, a long-term follow-up study showed that the compliance with the 6-hour early goal-directed resuscitation bundle returned to the baseline value, while the compliance with the 24-hour sepsis management bundle remained stable.[10] Considering the impact of the 6-hour early goal-directed resuscitation bundle and the 24-hour sepsis management bundles on clinical outcomes, use of the resuscitation bundle seems to be more strongly associated with clinical outcomes.[4] We designed this before-and-after study to test the hypothesis that a biweekly repetitive educational program for the emergency de- 
partment (ED) and ICU residents, nurses, and faculties led by a multidisciplinary team could improve the compliance rate for the sepsis resuscitation bundle and further reduce the mortality of severe sepsis and septic shock patients.

\section{Materials and Methods}

This before-and-after study was approved by the Institutional Review Board (IRB) of Severance Hospital (IRB No 4-20090295), and the need for informed consent was waived by the IRB. Adult patients aged 18 years or more who were admitted to the ED and diagnosed with severe sepsis/septic shock during a 20-month period after initiation of educational sessions were included as the intervention group (between November 2007 and July 2009). For the control group, patients who were admitted to the ED and diagnosed with severe sepsis/septic shock under the same criteria between January 2006 and September 2007 were included. The clinical data were retrospectively collected by reviewing the electronic medical records (EMR) of the patients. Severe sepsis was defined as sepsis associated with acute organ dysfunction. The term organ dysfunction was defined by the SOFA scoring system.[11] Each subgroup scoring higher than 3 was defined as specific organ failure. Septic shock was defined as acute circulatory failure (systolic blood pressure less than 90 $\mathrm{mmHg}$ despite volume resuscitation).[12] We excluded 22 patients who were younger than 18 years, pregnant, with trauma, readmitted within 1 week due to previous diagnosis of sepsis, transferred from another institution, or had a do-not-resuscitate order or a previous history of heart failure. We compared clinicians' adherence to the SSC guidelines for the implementation of the early goal-directed resuscitation bundle and clinical outcomes of the patients, including 28-day mortality, between the two groups.

\section{1) Intervention to improve adherence to the SSC guidelines}

The intervention consisted of two components; a multidisciplinary team and repeated education sessions. The multi-disciplinary team was organized under the participation of the faculties and nurses working in the ICU and ED. Physicians' subspecialties included emergency medicine, pulmonology, infectious disease, and anesthesiology. The multidisciplinary team provided 2-hour educational sessions during the study period, twice a month, starting in November 2007. The biweekly education sessions encompassed the definition of sepsis, components of the resuscitation bundle, and clinical evidence provided by the recent literature. They also included monthly feedback from the data of the previous month on the basis of the plan-do-study-act process.

\section{2) Management protocol}

In the intervention group we managed the patients by following the protocol based on the 6-hour early goal-directed resuscitation bundle.[13] The hemodynamic goal (mean arterial pressure $[\mathrm{MAP}]>65 \mathrm{mmHg}$ and central venous pressure [CVP] $>8 \mathrm{mmHg}$ ) was achieved with fluid resuscitation and vasopressor infusion. Dobutamine administration or transfusion of packed red blood cells were used to maintain central venous oxygen saturation $\left(\mathrm{ScvO}_{2}\right)$ over $70 \%$. We also intravenously administered initial empirical antimicrobial therapy within the first 3 hours of diagnosis after appropriate cultures were obtained. Initial empirical anti-infective therapy should include one or more drugs following the 2004 SSC guidelines. Antibiotics selection should be largely determined by institutional guidelines considering the source or focus of infection, the patient's immunologic status, and whether the infection is nosocomial or community-acquired. The 2004 SSC guidelines also recommend taking into consideration the institution's specific bacterial resistance patterns. If the patient needed ventilator care due to acute lung injury or acute respiratory distress syndrome, we applied mechanical ventilation using a lung protective strategy including low tidal volume of $6 \mathrm{ml} / \mathrm{kg}$ of predicted body weight, plateau airway pressure less than $30 \mathrm{~cm}$ $\mathrm{H}_{2} \mathrm{O}$, and application of positive end expiratory pressure table of acute respiratory distress syndrome network.

\section{3) Data collection and outcome measures}

Demographic data including age, gender, APACHE II score, origin of infection, and organ dysfunction were collected. To evaluate the compliance rate of the early goal-directed resuscitation bundle, we investigated the following items: blood culture before antibiotics, intravenous broad-spectrum antibiotics, and hemodynamic goals including $\mathrm{MAP} \geq 65 \mathrm{mmHg}$, $\mathrm{CVP} \geq 8 \mathrm{mmHg}$, and $\mathrm{ScvO}_{2} \geq 70 \%$.

The primary end point was the comparison of compliance rates for the early goal-directed resuscitation bundle for severe sepsis/septic shock patients during the first 6 hours after the diagnosis, between the two groups. We also compared 28-day mortality and length of stay (LOS) in the ICU and hospital. To evaluate the improvement in the achievement rate of the early goal-directed resuscitation bundle after the intervention process, we compared the rate of measurement of the serum lactate level, 
insertion of the central venous catheter, insertion of the arterial line, measurement of CVP and $\mathrm{ScvO}_{2}$, and appropriate use of antibiotics. We also compared the time interval from the diagnosis to the start of the monitoring and achievement of the goals. The goals consisted of administering antibiotics within 3 hours of the onset, and achievement of CVP, MAP, and $\mathrm{ScvO}_{2}$ goals within 6 hours.

\section{4) Statistical analysis}

To compare continuous data, Student's $t$-test or MannWhitney test was used. Categorical data were analyzed using the chi-squared test. Mortality was analyzed with the log-rank test and Breslow test, represented by Kaplan-Meier curves. KaplanMeier analysis was used to compare LOS in the ICU or hospital and mortality within 28 days.

\section{Results}

The intervention group had 87 patients and the control group had 88 patients. Age, sex, and APACHE II score were com- parable between the two groups (Table 1). At time-point zero, there were no significant differences in the origin of the infection between the groups. In the study population, the most common origin of sepsis was pneumonia and abdominal infection. The prevalence of organ dysfunction was comparable between the groups. The most common major organ dysfunctions were cardiovascular and respiratory dysfunction.

Considering each of the resuscitation bundle items, antibiotics were more frequently given within 3 hours in the intervention group ( $85 \%$ vs. $41 \%, \mathrm{p}<0.001)$. The rate of achieving hemodynamic goals was also higher in the intervention group (CVP 83\% vs. 27\%, p < 0.001; MAP 98\% vs. 89\%, p = 0.013; CVP and MAP, $94 \%$ vs. $25 \%$, p < 0.001). $\mathrm{ScVO}_{2}$ level was incommensurable because it was not measured in the control group. The rate of patients who achieved all hemodynamic goals within 6 hours after diagnosis $(80 \%$ vs. $0 \%, \mathrm{p}<0.001)$ was definitely higher in the intervention group (Table 2).

The time from the diagnosis to the achievement of variables of the early goal-directed resuscitation bundle were compared between the two groups. After completing the training program,

Table 1. Demographic data, origin of infection, and organ dysfunction

\begin{tabular}{lccc}
\hline \hline & Intervention $(\mathrm{n}=87)$ & Control (n=88) & $\mathrm{p}$ value \\
\hline APACHE II & $20.0 \pm 6.6$ & $21.4 \pm 5.7$ & 0.133 \\
Age, yr & $64.3 \pm 12.3$ & $64.7 \pm 14.7$ & $44(50)$ \\
Male & $46(53)$ & & 0.847 \\
Origin of infection & & $30(34)$ & 0.818 \\
$\quad$ Pneumonia & $33(38)$ & $21(24)$ & 0.828 \\
Acute abdomen & $20(23)$ & $18(21)$ & 0.888 \\
UTI & $13(15)$ & $1(1)$ & 0.695 \\
Meningitis & $2(2)$ & $4(5)$ & 0.313 \\
Soft tissue infection & $7(8)$ & $8(9)$ & 0.215 \\
Others & $10(12)$ & $5(6)$ & 0.702 \\
$\quad$ Multiple infection sites & $3(3)$ & & 0.384 \\
Organ dysfunction & & $66(75)$ & \\
Cardiovascular & $66(76)$ & $59(67)$ & 0.966 \\
Respiratory & $48(55)$ & $23(48)$ & 0.145 \\
Renal & $28(32)$ & $21(24)$ & 0.475 \\
Hepatic & $15(17)$ & $16(18)$ & 0.369 \\
Coagulation & $9(10)$ & $15(17)$ & 0.206 \\
Neurologic & $10(12)$ & 0.203 \\
\hline
\end{tabular}

Values are presented as mean $\pm \mathrm{SD}$ or $\mathrm{n}(\%)$. ${ }^{*}$ Organ dysfunction was defined by SOFA scoring greater than 3 . APACHE II: acute physiology and chronic health evaluation; UTI: urinary tract infection.

Table 2. Compliance to sepsis resuscitation bundle within 6 hours from diagnosis

\begin{tabular}{|c|c|c|c|}
\hline & Intervention $(\mathrm{n}=87)$ & Control $(n=88)$ & $\mathrm{p}$ value \\
\hline Antibiotics administered within $3 \mathrm{~h}$ & $74(85)$ & $36(41)$ & $<0.001$ \\
\hline Blood culture before antibiotics & $84(97)$ & $79(90)$ & 0.140 \\
\hline $\mathrm{CVP} \geq 8 \mathrm{mmHg}$ & $72(83)$ & $24(27)$ & $<0.001$ \\
\hline $\mathrm{MAP} \geq 65 \mathrm{mmHg}$ & $86(99)$ & 78 (89) & 0.013 \\
\hline All measured goals & $70(80)$ & $0(0)$ & $<0.001$ \\
\hline
\end{tabular}

Values are presented as mean $\pm \mathrm{SD}$ or $\mathrm{n}(\%)$. CVP: central venous pressure; MAP: mean arterial pressure. 


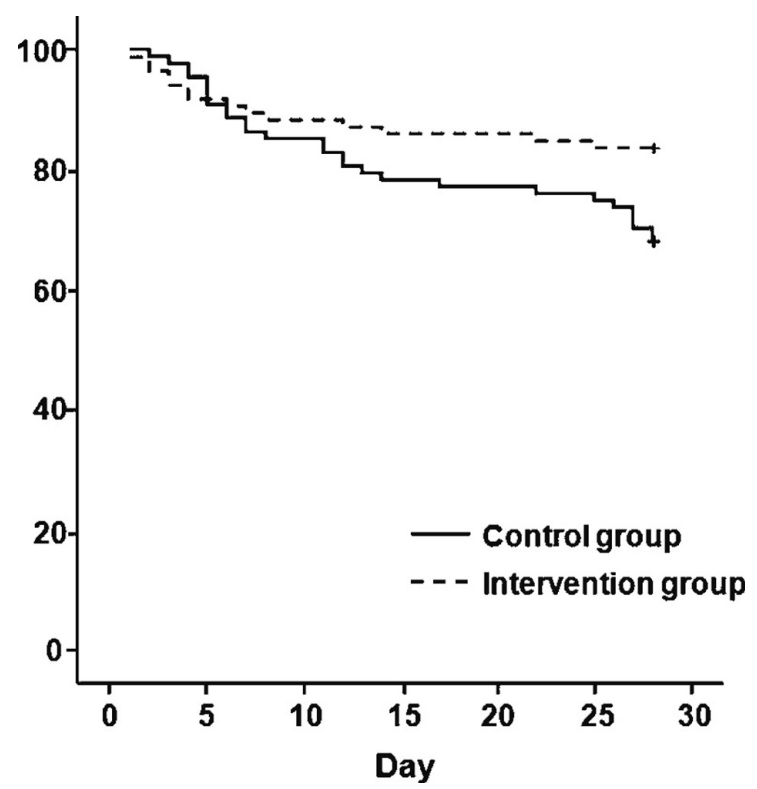

Fig. 1. Comparison of the 28-day survival rate between the intervention and control groups. Kaplan-Meier curves depicting the probability of survival of patients with severe sepsis or septic shock in the intervention group and the control group according to the length of survival $(\mathrm{p}=0.04$ by Breslow test $)+$ means censored data.

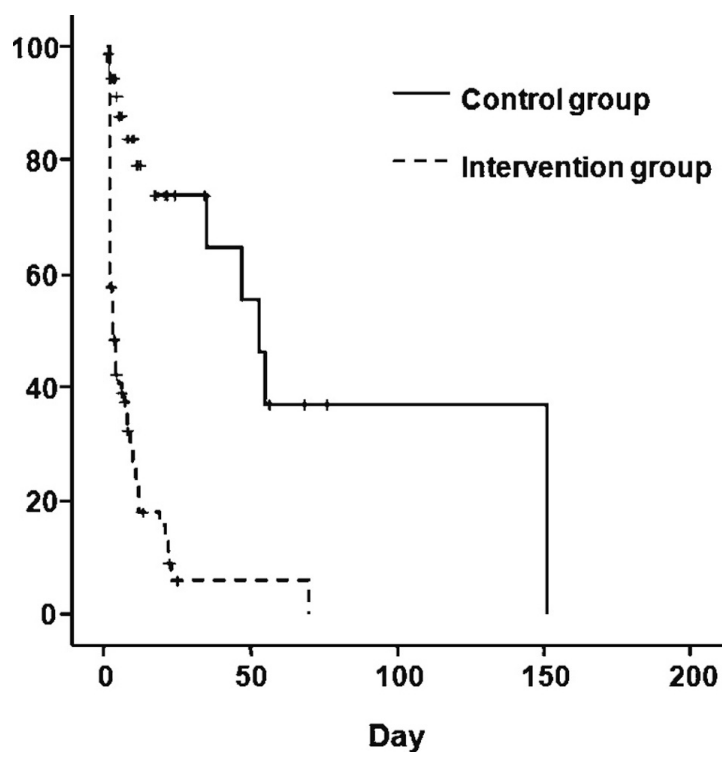

Fig. 2. Comparison of LOS in the ICU between the intervention and control groups. Kaplan-Meier curves depict the probability of remaining in the ICU for patients with severe sepsis or septic shock in the intervention group and the control group according to the LOS in the ICU ( $p=0.001$ by Log Rank test $)+$ means censored data. LOS: length of stay; ICU: intensive care unit.

the times to administering antibiotics, insertion of central venous catheter, and achievement of CVP and MAP goals were significantly reduced (118 vs. 245 min, p < 0.001; 40 vs. 122 $\min , p=0.0007 ; 135$ vs. $197 \min , p=0.0163 ; 91$ vs. $179 \min , p$

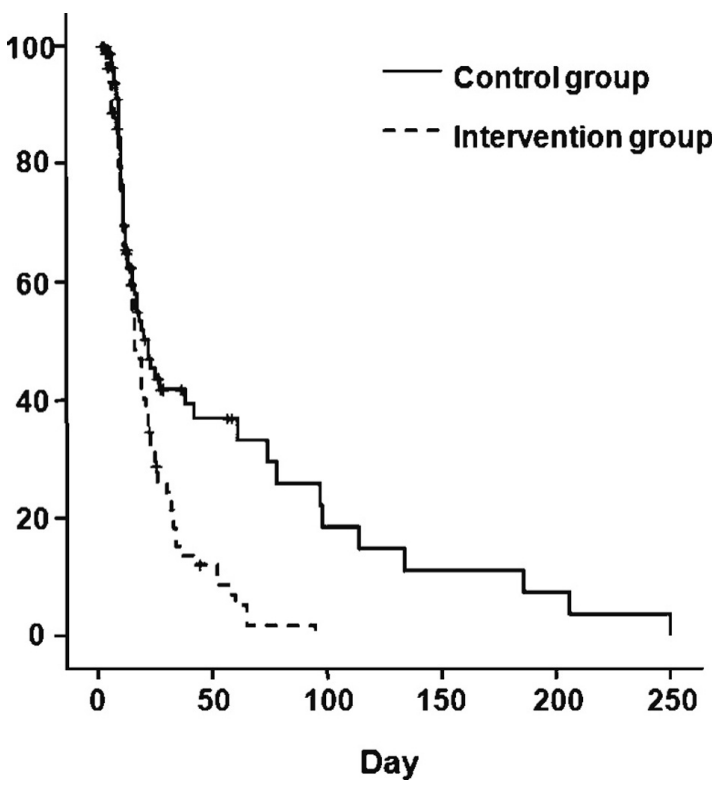

Fig. 3. Comparison of the number of days in hospital between the intervention and the control groups. Kaplan-Meier curves depict the probability of remaining in the hospital for patients with severe sepsis or septic shock in the intervention group and the control group according to the LOS in hospital ( $\mathrm{p}=0.078$ by Breslow test) + means censored data. LOS: length of stay.

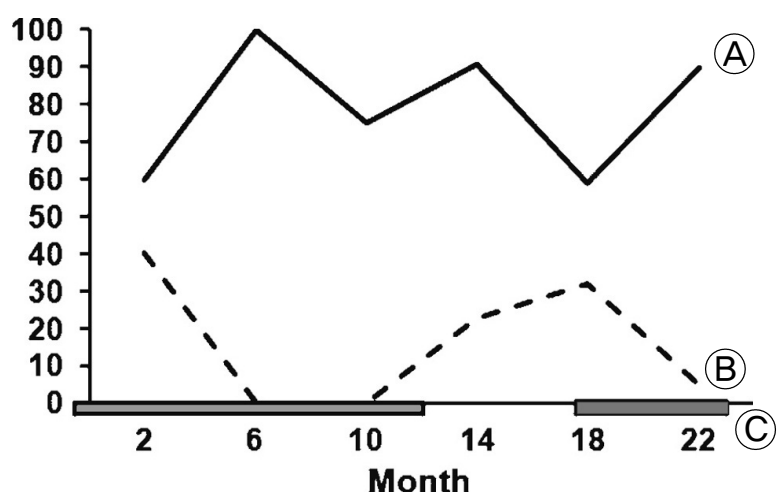

Fig. 4. Relationship between training and outcome. The x-axis shows the 22-month study period divided into 4-month intervals comparing the goal achievement rates between each period, and the $\mathrm{y}$-axis shows the 28-day mortality rate (A) and the achievement rate of the early goal-directed resuscitation bundle (B). C indicates the time period when training was carried out.

$<0.001$, respectively)

The patients in the intervention group showed a significantly lower mortality rate at 28 days after the diagnosis (16\% vs. $32 \%$, $p=0.04)$ than those in the control group. Kaplan-Meier plots of the probability of remaining alive are shown in Fig. 1. The Breslow test showed statistical significance in the difference in 28-day mortality between the groups (Fig. 1, p = 0.040). LOS in the ICU analyzed by Kaplan-Meier test was significantly longer in the control group (Fig. 2, p=0.001), while LOS in the hospital 
was not significantly different between the two groups (Fig. 3, p $=0.078$ ). Discontinuation of the training for 4 months reduced the goal achievement rate and increased the 28-day mortality rate, although they were restored after resuming the program (Fig. 4).

In the intervention group, 17 patients did not have their resuscitation goals completed, while the remaining 70 patients did. There was a statistically significant difference in the 28-day mortality rate in the patients who did or did not complete the bundle ( $11 \%$ vs. $41 \%, \mathrm{p}=0.004)$, while LOS in the ICU and LOS in the hospital were not significantly different according to the bundle completion rate. $(\mathrm{p}=0.062)$

Norepinephrine $(53 \%$ vs. $34 \%, \mathrm{p}=0.019)$ was more frequently administered in the intervention group, compared with the control group. Fewer patients received dopamine (50\% vs. $91 \%, \mathrm{p}<0.001)$ and dobutamine (4\% vs. $19 \%, \mathrm{p}=0.006)$ in the intervention group, respectively. Steroids were more frequently administered in the intervention group, compared with the control group ( $34 \%$ vs. $19 \%, \mathrm{p}=0.036)$, and there was no significant difference in the use of vasopressin $(14 \%$ vs. $7 \%$, p = $0.138)$.

\section{Discussion}

The present study showed that quality improvement action including repeated education provided by a multidisciplinary team could improve the adherence to SCC guidelines, thereby improving clinical outcomes in severe sepsis and septic shock patients. The adherence rate to the SCC guidelines before education or quality improvement actions was poor, a finding consistent with previous studies.[10,14] In our study, a specially organized multi-disciplinary team with good collaboration and a training program improved the management process of severe sepsis and septic shock patients. Trzeciak et al.[9] showed that effective emergency medicine and collaborative critical care improved the early achievement rate of goal-directed therapy. Our collaborative sepsis management team showed improved compliance and outcomes.

A nation-wide study demonstrated that a training program based on the SCC guidelines improved compliance with the recommended processes of care for severe sepsis in the ICU.[10,15] However, Ferre also showed a discrepancy in adherence to the protocol for the early goal-directed resuscitation bundle and the 24-hour management bundle. In this study, compliance with the protocol for the sepsis management bundle was maintained even after the 1-year follow-up was finished, while the compliance for the early goal-directed resuscitation bundle returned to the baseline level.

In the intervention group, we found a significant relationship between the achievement rate of the early goal-directed resuscitation bundle as recommended by the SCC guidelines and the mortality rate. Recent studies showed that non-compliance with the 6-hour sepsis resuscitation bundle increased mortality while non-compliance with the 24-hour sepsis management bundle did not.[4]

Studies have been conducted on the effect of education in clinical fields and reports have revealed the effect of education on the behavior of the medical staff.[16] Ordinary didactic approaches such as printed materials or practice guidelines for health care providers, including physicians, failed to change their behavior.[17] Only evidence-based educational approaches are known to have an impact on health care providers' behavior. Such approaches include interactive educational activities, sequenced and multifaceted interventions, educational outreach or academic detailing, and audit and feedback to physicians.[18,19] Constant re-educational programs were essential for the successful implementation of the sepsis bundle.

Standardization and protocolization are important for improving clinical practice.[20] Many researchers tried to demonstrate that implementation of an evidence-based management protocol improved clinical outcomes of sepsis patients, but their results were not consistent.[5-7,9,14,21] Most of the trials used more rigorous fluid management earlier in the treatment protocol, more appropriate antibiotics therapy, and shorter LOS in the ICU or hospital, and the effects of the tested interventions on mortality varied. A few studies demonstrated mortality benefits,[6,22] while others did not.[21] These inconsistent results were thought to be related to clinicians' adherence to the protocol. Nguyen et al. demonstrated that the completion of early goal-directed therapy was significantly associated with lower mortality[7] (odds ratio, 0.36; 95\% confidence interval, 0.17$0.79 ; \mathrm{p}=0.01)$. Compared to previous studies, the completion rate after intervention in our study was relatively high. This high rate might result from the fact that we organized the clinical pathway with a periodic survey of the treatment process and continuous provision of training sessions.

The time to administration of broad-spectrum antibiotics was improved after intervention in this study, while the administration rate itself did not show significant changes. Similar improvement in earlier administration of antimicrobial agents was noticed in several recent studies.[21].

Among vasopressors, dopamine and norepinephrine are most 
frequently used, and are recommended by the SCC guidelines. In our study, there was a noticeable change in the use of vasopressors. More vasopressin and less dopamine were used in the intervention group. This change was thought to result from the physicians' concerns about the arrhythmogenicity of dopamine and relative deficiency of vasopressin.[23,24] In addition, during the study periods, more physicians would tend to use steroids in patients with septic shock who, despite adequate fluid replacement, required vasopressor therapy to maintain adequate blood pressure. Therefore, steroid use showed a significant difference between the two groups in the course of time (34\% vs. $19 \%, \mathrm{p}=0.036)$.

As demonstrated by Rivers,[22] time-dependant characteristics of sepsis management required involved clinicians to make standardized protocols, but its multi-disciplinary nature made implementation of protocols challenging. The present study confirmed that the sepsis resuscitation bundle, as well as the treatment bundle, could be implemented and maintained by continuously conducting surveys and education, and benefit sepsis patients with standardized care and early admission to the ICU.

Limitations of this study are as follows. Since creating a multi-disciplinary team, provision of repeated training, and formulating a resuscitation bundle implement pathway were simultaneously carried out, we could not differentiate the impacts of each factor. Moreover, since the control group data were retrospectively collected, randomization could not be performed. However, since all patients who were admitted to the ED and diagnosed as having severe sepsis/septic shock between January 2006 and September 2007 were allocated to the control group, selection bias would be minimal. Random allocation of patients to the intervention group would not be ethical, so the comparison between the intervention and control groups could be performed only observationally. In our institution, moreover, since patients' data were recorded in the EMR system according to the institutional guidelines, we gathered all parameters relevant to the resuscitation bundle and patients' outcome. Another important limitation was that we did not know how long the effect of training could be maintained and we could not confirm the ideal interval between training sessions. However, discontinuation of the training program for 4 months reduced the goal achievement rate and increased the 28-day mortality rate, although the goal achievement rate and mortality rate were restored after resuming the training (Fig. 4). We would like to suggest that repeated training with an interval between training sessions of at least 2 or 3 months is necessary to maintain improved com- pliance and clinical outcomes. The other limitation was that our hypothesis was based upon a premise that we could exclude the effect of the 24-hour sepsis management bundle on the clinical outcome. However, compliance with the 6-hour early goal-directed resuscitation bundle was in connection with the 24-hour sepsis management bundle.

In conclusion, we demonstrated that implementation of an evidence-based management protocol for severe sepsis or septic shock patients improved clinical outcomes. Repeated exposure to an educational program and interdisciplinary communication was helpful to maintain a high completion rate for the management bundles, which in turn decreased ICU mortality in sepsis patients.

\section{References}

1) Brun-Buisson C, Doyon F, Carlet J, Dellamonica P, Gouin F, Lepoutre A, et al: Incidence, risk factors, and outcome of severe sepsis and septic shock in adults. A multicenter prospective study in intensive care units. French ICU Group for Severe Sepsis. JAMA 1995; 274: 968-74.

2) Guidet B, Aegerter P, Gauzit R, Meshaka P, Dreyfuss D: CUB-Réa Study Group: Incidence and impact of organ dysfunctions associated with sepsis. Chest 2005; 127: 942-51.

3) Dellinger RP, Levy MM, Rhodes A, Annane D, Gerlach H, Opal SM, et al: Surviving sepsis campaign: international guidelines for management of severe sepsis and septic shock: 2012. Crit Care Med 2013; 41: 580-637.

4) Gao F, Melody T, Daniels DF, Giles S, Fox S: The impact of compliance with 6-hour and 24-hour sepsis bundles on hospital mortality in patients with severe sepsis: a prospective observational study. Crit Care 2005; 9: R764-70.

5) Kortgen A, Niederprüm P, Bauer M: Implementation of an evidence-based "standard operating procedure" and outcome in septic shock. Crit Care Med 2006; 34: 943-9.

6) Micek ST, Roubinian N, Heuring T, Bode M, Williams J, Harrison C, et al: Before-after study of a standardized hospital order set for the management of septic shock. Crit Care Med 2006; 34: 2707-13.

7) Nguyen HB, Corbett SW, Steele R, Banta J, Clark RT, Hayes $\mathrm{SR}$, et al: Implementation of a bundle of quality indicators for the early management of severe sepsis and septic shock is associated with decreased mortality. Crit Care Med 2007; 35 : 1105-12.

8) Rivers E: Implementation of an evidence-based "standard operating procedure" and outcome in septic shock: what a 
sepsis pilot must consider before taking flight with your next patient. Crit Care Med 2006; 34: 1247.

9) Trzeciak S, Dellinger RP, Abate NL, Cowan RM, Stauss M, Kilgannon JH, et al: Translating research to clinical practice: a 1-year experience with implementing early goal-directed therapy for septic shock in the emergency department. Chest 2006; 129: 225-32.

10) Ferrer R, Artigas A, Levy MM, Blanco J, González-Díaz G, Garnacho-Montero J, et al: Improvement in process of care and outcome after a multicenter severe sepsis educational program in Spain. JAMA 2008; 299: 2294-303.

11) Vincent JL, Moreno R, Takala J, Willatts S, De Mendonça A, Bruining H, et al: The SOFA (Sepsis-related Organ Failure Assessment) score to describe organ dysfunction/failure. On behalf of the working group on sepsis-related problems of the European Society of Intensive Care Medicine. Intensive Care Med 1996; 22: 707-10.

12) Bone RC, Balk RA, Cerra FB, Dellinger RP, Fein AM, Knaus WA, et al: Definitions for sepsis and organ failure and guidelines for the use of innovative therapies in sepsis. The ACCP/SCCM Consensus Conference Committee. American College of Chest Physicians/Society of Critical Care Medicine. 1992. Chest 2009; 136(5 Suppl): e28.

13) Dellinger RP, Carlet JM, Masur H, Gerlach H, Calandra T, Cohen J, et al: Surviving Sepsis Campaign guidelines for management of severe sepsis and septic shock. Crit Care Med 2004; 32: 858-73.

14) De Miguel-Yanes JM, Andueza-Lillo JA, González-Ramallo VJ, Pastor L, Muñoz J: Failure to implement evidence-based clinical guidelines for sepsis at the ED. Am J Emerg Med 2006; 24: 553-9.

15) Chen YC, Chang SC, Pu C, Tang GJ: The impact of nationwide education program on clinical practice in sepsis care and mortality of severe sepsis: a population-based study in
Taiwan. PLoS One 2013; 8: e77414.

16) Paes BA, Modi A, Dunmore R: Changing physicians' behavior using combined strategies and an evidence-based protocol. Arch Pediatr Adolesc Med 1994; 148: 1277-80.

17) Gray J: Changing physician prescribing behaviour. Can J Clin Pharmacol 2006; 13: e81-4.

18) Davis D, O'Brien MA, Freemantle N, Wolf FM, Mazmanian $\mathrm{P}$, Taylor-Vaisey A: Impact of formal continuing medical education: do conferences, workshops, rounds, and other traditional continuing education activities change physician behavior or health care outcomes? JAMA 1999; 282: 867-74.

19) Davis DA, Thomson MA, Oxman AD, Haynes RB: Changing physician performance. A systematic review of the effect of continuing medical education strategies. JAMA 1995; 274: 700-5.

20) Shin HJ, Lee KH, Hwang SO, Kim H, Shin TY, Kim SC: The efficacy of early goal-directed therapy in septic shock patients in the emergency department: severe sepsis campaign. Korean J Crit Care Med 2010; 25: 61-70.

21) De Miguel-Yanes JM, Muñoz-González J, Andueza-Lillo JA, Moyano-Villaseca B, González-Ramallo VJ, BustamanteFermosel A: Implementation of a bundle of actions to improve adherence to the surviving sepsis campaign guidelines at the ED. Am J Emerg Med 2009; 27: 668-74.

22) Rivers E, Nguyen B, Havstad S, Ressler J, Muzzin A, Knoblich B, et al: Early goal-directed therapy in the treatment of severe sepsis and septic shock. N Engl J Med 2001; 345: 1368-77.

23) Lauzier F, Lévy B, Lamarre P, Lesur O: Vasopressin or norepinephrine in early hyperdynamic septic shock: a randomized clinical trial. Intensive Care Med 2006; 32: 1782-9.

24) Sharshar T, Blanchard A, Paillard M, Raphael JC, Gajdos P, Annane D: Circulating vasopressin levels in septic shock. Crit Care Med 2003; 31: 1752-8. 